\title{
Saturation momentum scale extracted from semi-inclusive transverse spectra in high-energy $p p$ collisions
}

\author{
Takeshi Osada* \\ Department of Physics, Faculty of Liberal Arts and Sciences, and Tokyo City University, \\ Tamazutsumi 1-28-1, Setagaya-ku, Tokyo 158-8557, Japan \\ Takuya Kumaoka \\ Department of Physics, Shinshu University, Matsumoto 390-8621, Japan
}

(Received 24 April 2019; revised manuscript received 20 August 2019; published 26 September 2019)

\begin{abstract}
Geometric scaling is well confirmed for transverse-momentum distributions observed in proton-proton collisions at Large Hadron Collider (LHC) energies. We introduced multiplicity dependence on a saturation momentum of the geometrical scaling, assuming the scaling holds for semi-inclusive distributions as well as for inclusive distributions. The saturation momentum is usually given by Bjorken's $x$ variable, but redefinition of the scaling variable can make the saturation momentum a function of collision energy $W$. We treat the energy as a free parameter (denoted $W^{*}$ to distinguish it from $W$ ) and associate the energy-dependent saturation momentum $Q_{\text {sat }}\left(W^{*}\right)$ with particle number density. By using $Q_{\text {sat }}\left(W^{*}\right)$ for a scaling variable $\tau$, we show semi-inclusive distributions can be geometrically scaled, i.e., all semi-inclusive spectra observed at $W=0.90,2.76$, and $7.00 \mathrm{TeV}$ overlap one universal function. The particle density dependencies of mean transverse momentum $\left\langle p_{T}\right\rangle$ for LHC energies scales in terms of $Q_{\text {sat }}\left(W^{*}\right)$. Furthermore, our model explains a scaling property of event-by-event $p_{T}$ fluctuation measure $\sqrt{C_{m}} /\left\langle p_{T}\right\rangle$ at LHC energies for $p p$ collisions, where $C_{m}$ is a two-particle transverse-momentum correlator. Our analysis of the $p_{T}$ fluctuation makes possible to evaluate a nonperturbative coefficient of the gluon correlation function.
\end{abstract}

DOI: 10.1103/PhysRevC.100.034906

\section{INTRODUCTION}

Studies of small collision systems in high-multiplicity events is attracting considerable interest [1] because of the collective phenomena which attribute to the formation of a strongly interacting collectively expanding quark-gluon medium [2-5]. A remarkable similarity has been observed between strange particles production in $p p$ collisions and that in $\mathrm{Pb}-\mathrm{Pb}$ collisions, suggesting the possibility of deconfined QCD phase formation in small systems [6]. In such $p p$ collisions, the charged particle pseudorapidity density rises as a power of energy $[7,8]$, which can be explained by the theory of gluon saturation $[9,10]$. Recombination of gluons [11] in high-particle-number density state causes the saturation, and the gluon distribution function ceases growing from some intrinsic scale of the transverse momentum $Q_{s}$ [12]. The color glass condensate (CGC) [13-15] is an effective theory to describe saturated gluons with small $x$ as classical color fields radiated by color sources at higher rapidity. The existence of

*t-osada@tcu.ac.jp

Published by the American Physical Society under the terms of the Creative Commons Attribution 4.0 International license. Further distribution of this work must maintain attribution to the author(s) and the published article's title, journal citation, and DOI. Funded by $S C O A P^{3}$.
$Q_{s}$ which separates the degree of freedom into fast frozen color sources and slow dynamical color fields [16] is the underlying assumption of the effective theory. The scaling of the limiting fragmentation curves [17] is one of the crucial pieces of evidence for the picture of the CGC $[18,19]$.

Another experimental evidence of CGC hypothesis is a geometrical scaling $[20,21]$ (GS) confirmed originally in results on total $\gamma^{*} p$ cross section [22]. A term of the "geometrical" of this GS comes from that survival probability of a color dipole [23-25] is determined by the geometric relationship between the dipole size and the saturation radius given by $Q_{s}^{-1}(x)[16,26]$, where $x$ is a Bjorken variable. In this article, since we will deal with multiparticle production in the central rapidity region of high-energy $p p$ collisions, we have $x=$ $p_{T} / W$, where $p_{T}$ and $W$ are the transverse momentum and colliding energy of the incident proton, respectively. With $x_{0}$, $Q_{0}$, and $\lambda$ as constants (see, Sec. II for details), the saturation momentum is given by [27]

$$
Q_{s}(x) \equiv Q_{0}\left(\frac{x}{x_{0}}\right)^{-\lambda / 2} .
$$

If such momentum is the only scale that controls $p_{T}$ distribution, then it should exhibit GS behavior; i.e., when one normalizes inclusive transverse-momentum spectra observed with an appropriate constant $S_{T}$ (interpreted later as reaction effective transverse cross-sectional area), the data points lie on a characteristic curve $\mathcal{F}(\tau)$ which is only depends on the 
scaling variable $\tau \equiv p_{T}^{2} / Q_{s}^{2}$ and the curve does not depend on $W$. In particular, the scaling property has been vigorously studied for $p p$ collisions obtained at the Large Hadron Collider (LHC) energies [28-31] and GS is observed in single inclusive distributions of charged hadrons [28] and recently observed direct photons from heavy-ion collisions [32]. Since $Q_{s}(x)$ includes $p_{T}$ dependence via Bjorken $x$; i.e.,

$$
Q_{s}\left(\frac{p_{T}}{W}\right)=Q_{0}\left(x_{0} W\right)^{\frac{\lambda}{2}} p_{T}^{-\frac{\lambda}{2}},
$$

we unify the terms of $p_{T}$ contained in $\tau$ and redefine the rest that depends on $W$ as an energy-dependent saturation momentum $Q_{\text {sat }}(W)$ [33]. Namely, the scaling variable can be rewritten as

$$
\begin{aligned}
\tau & =\left[\frac{p_{T}}{Q_{\text {sat }}(W)}\right]^{2+\lambda}, \\
Q_{\text {sat }}(W) & \equiv Q_{0}\left(\frac{x_{0} W}{Q_{0}}\right)^{\frac{\lambda}{\lambda+2}},
\end{aligned}
$$

and the GS is expressed as [34]

$$
\frac{1}{S_{T}} \frac{d^{2} N_{\mathrm{ch}}}{d p_{T}^{2} d y}=\mathcal{F}(\tau)
$$

where $\mathcal{F}(\tau)$ is a so-called universal function of GS. Under an assumption that a local parton hadron duality [35] as a hadronization model is appropriate, the particle density at the central rapidity region $(y \approx 0)$ relates to $Q_{\text {sat }}(W)$ as follows:

$$
\left\langle\frac{d N_{\mathrm{ch}}}{d y}\right\rangle \propto S_{T} Q_{\mathrm{sat}}^{2}(W),
$$

where $\langle\cdots\rangle$ denotes the average over single inclusive distribution (or over minimum bias events). Since the particle number density is known to increases gradually with collision energy $W$, we expect $Q_{\text {sat }}(W)$ to also increases gradually with $W$.

Let us suppose that GS holds not only for inclusive distributions but also for the semi-inclusive distributions, i.e., inclusive distribution with fixed multiplicity or limited multiplicity class [36,37]. For the semi-inclusive spectra $\frac{d^{2} n_{\mathrm{ch}}}{d p_{T}^{2} d y}$, as the case of inclusive one, we assume that there exists a saturation momentum for the spectrum classified by multiplicity as well and we propose to represent it by effective energy $W^{*}$; i.e.,

$$
\frac{1}{S_{T}^{*}} \frac{d^{2} n_{\mathrm{ch}}}{d p_{T}^{2} d y}=\mathcal{F}(\tau)
$$

where, instead of Eq. (3a), we use

$$
\tau=\left[\frac{p_{T}}{Q_{\text {sat }}\left(W^{*}\right)}\right]^{2+\lambda} .
$$

It should be noted here that the universal function $\mathcal{F}$ in Eq. (5a) is the same as that in Eq. (3c). Here $S_{T}^{*}$ and $W^{*}$ are determined to reproduce the spectrum obtained by the experiment. In particular, this $W^{*}$ is a fit parameter introduced replacing the actual beam energy $W$ in Eq. (3b). Hence, we intend to check whether GS found in inclusive distribution is restored even in semi-inclusive distribution.
It may be appropriate to give some explanations for $W^{*}$ here. As discussed in detail later in Sec. II, the energydependent saturation momentum $Q_{\text {sat }}$ gives a typical scale of transverse momentum $p_{T}$. That is, $Q_{\text {sat }}$ is the solution $p_{T}$ of an equation $Q_{s}\left(p_{T} / W\right)=p_{T}$ for each colliding energy $W$. Because $Q_{\text {sat }}$ itself is a scale of transverse momentum, the inverse of it is a typical transverse size scale of saturated gluons. Hence as seen in Eq. (4), the ratio of effective interaction cross-sectional area $S_{T}$ to the cross-sectional area per gluon $Q_{\text {sat }}^{-2}$ governs the mean charged particle density of the inclusive distribution. On the other hand, for semi-inclusive collisions classified by multiplicity, $S_{T}^{*}$ and $Q_{\text {sat }}\left(W^{*}\right)$ should be related to each other by the constraint of the fixed multiplicity. We will discuss the relation between $Q_{\text {sat }}\left(W^{*}\right)$ and $S_{T}^{*}$ of the semi-inclusive distribution in some detail in Sec. III and also comment on the physical meaning of $W^{*}$.

This article is organized as follows. In Sec. II, we briefly review GS hypothesis and we confirm that it holds well for inclusive transverse spectra observed in $p p$ collisions at LHC energies. Then we determine the universal function of GS used throughout this article. In Sec. III, the effective energy $W^{*}$ is determined from the semi-inclusive transverse spectra. By using the scaling variables with $Q_{\text {sat }}\left(W^{*}\right)$, we show that the transverse-momentum spectra observed in the different multiplicity classes at the different collision energies scale to the universal function found in Sec. II. We also show that the multiplicity dependence of the mean transverse momentum scales with $Q_{\text {sat }}\left(W^{*}\right)$. Furthermore, we analyze the scaling behavior of a normalized fluctuation measure of transverse momentum and consider it as a result of the correlation between particles generated from color flux tubes. We close with Sec. IV containing the summary and some concluding remarks.

\section{GS FOR INCLUSIVE $p_{T}$ DISTRIBUTION}

The transverse-momentum spectra of various energies for $p p$ collisions never scale with variable $p_{T}$ because their intensities and slopes depend on the colliding energy $W$. However, for high-energy collisions in which the number of soft gluons inside the proton saturates, the transverse-momentum spectrum depends only on a scaling variable defined by Eq. (3a) with Eq. (3b). Let us examine the quantitative difference between $Q_{s}(x)$ and $Q_{\text {sat }}(W)$ at LHC energies. We show them as a function of $p_{T}$ in Fig. 1 for the case of $\lambda=0.22$, $x_{0}=1.0 \times 10^{-3}, Q_{0}=1.0 \mathrm{GeV} / c$ [34], and we will fix the values from now on. Since $Q_{s}$ is less dependent on $p_{T}$ for $p_{T} \gtrsim 0.5 \mathrm{GeV} / c$, one may use $Q_{\text {sat }}(W)$ instead of $Q_{s}$ as a typical momentum scale. The values of $Q_{\text {sat }}(W)$ obtained from inclusive $p_{T}$ spectra at energy $W=0.90,2.76,7.00 \mathrm{TeV}$ are $0.99,1.11$, and $1.21 \mathrm{GeV} / c$, respectively. As shown in Fig. 2, experimental data observed by the ALICE [38] and CMS Collaborations [39] suggests the validity of GS especially for $\tau^{1 /(2+\lambda)} \lesssim 10$. The curve emerging from a plot of the $p_{T}$ spectra with using the scaling variable $\tau$ can be fitted well by the so-called Tsallis-type function [40,41];

$$
\mathcal{F}(\tau)=\left[1+(q-1) \frac{\tau^{1 /(\lambda+2)}}{\kappa}\right]^{-1 /(q-1)},
$$




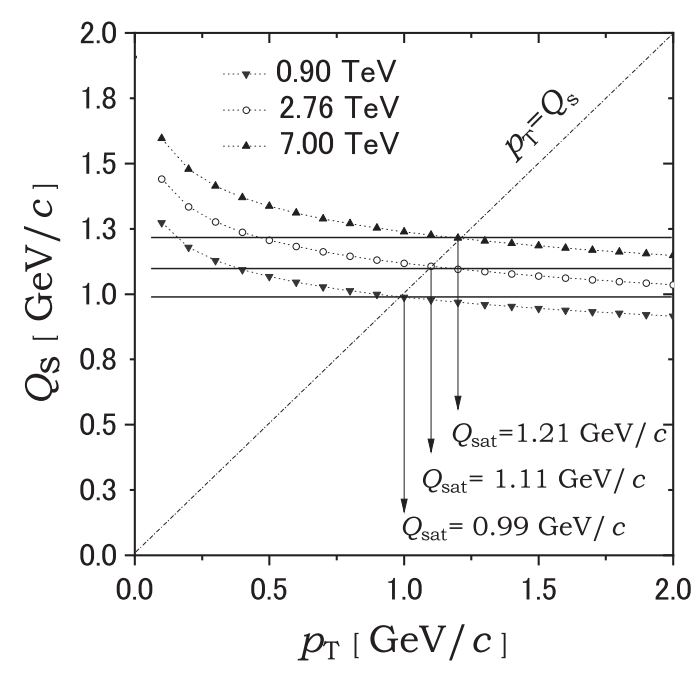

FIG. 1. Saturation momentum $Q_{s}$ (dotted curve with triangle and circle symbols) and energy-dependent saturation momentum $Q_{\text {sat }}$ (horizontal solid lines) for $W=0.90,2.76$, and $7.00 \mathrm{TeV}$. The longand-short-dashed line represents $Q_{s}=p_{T}$. The intersections of the line and the dotted curve give $Q_{\text {sat }}$ at each $W$. For $W=0.90,2.76$ and 7.00 TeV, $Q_{\text {sat }}=0.99,1.11,1.21 \mathrm{GeV} / c$, respectively.

where the nonextensive parameter $q=1.134$ and $\kappa=0.1293$ are used. The effective cross-sectional area in Eq. (3c) is determined as $S_{T}=22.66 \mathrm{GeV}^{-2}$. In this way, the transversemomentum distribution indeed exhibits GS behavior for $p p$ collisions in the LHC energies. It seems appropriate to shortly comment on the energy-dependent saturation momentum $Q_{\text {sat }}$ and an effective temperature $T_{\text {eff }}$ (or a slope parameter) $[29,42]$ here. In case of a Tsallis-type distribution function,

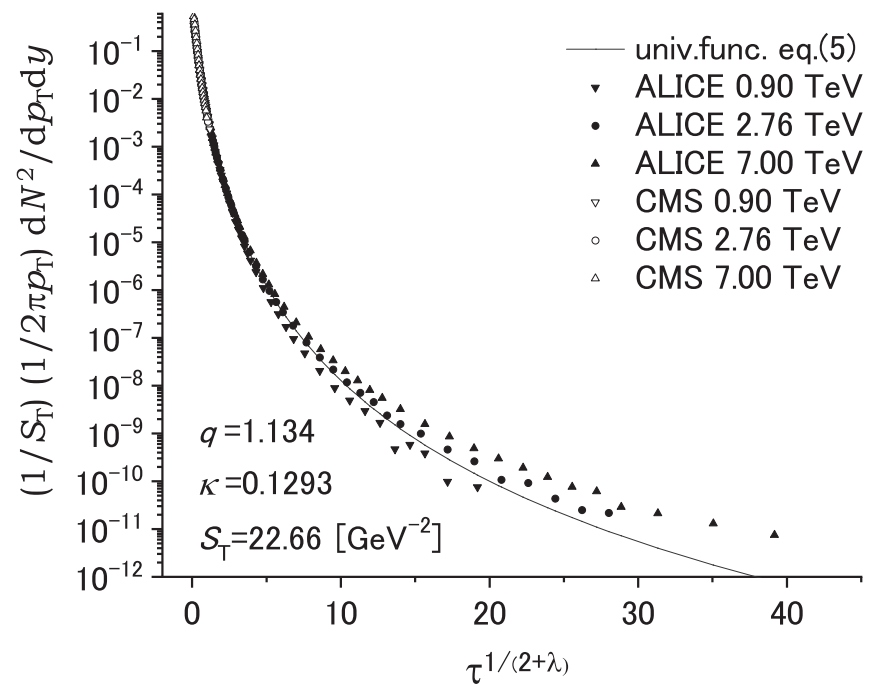

FIG. 2. The transverse-momentum distributions exhibit geometrical scaling behavior for $p p$ collisions at $W=0.90,2.76$, and $7.00 \mathrm{TeV}$. Experimental data (indicated by triangles or circles) are observed by the ALICE Collaboration [38] and CMS Collaboration [39]. The solid curve is the universal function $\mathcal{F}(\tau)$ with $q=1.134$, $\kappa=0.1293$, and $\lambda=0.22$ [see Eq. (6)]. The effective interaction cross-sectional area $S_{T}=22.66 \mathrm{GeV}^{-2}$ is used.
$T_{\text {eff }}$ can be defined as

$$
\frac{1}{2 \pi p_{T}} \frac{d^{2} N_{\mathrm{ch}}}{d p_{T} d y}=C\left[1+(q-1) \frac{p_{T}}{T_{\mathrm{eff}}}\right]^{-1 /(q-1)} .
$$

Here one may interpret the constant $C$ as $S_{T}$. Since the transverse spectra experimentally observed exhibits good GS behavior, the effective temperature $T_{\text {eff }}$ must have energy dependence to cancel the energy dependence of

$$
p_{T}=Q_{0}\left(\frac{x_{0} W}{Q_{0}}\right)^{\frac{\lambda}{\lambda+2}} \tau^{\frac{1}{2+\lambda}}
$$

which is obtained from Eq. (3a). Hence, the property of the GS determined the energy dependence of $T_{\text {eff }}$ and that $T_{\text {eff }}$ should be proportional to $Q_{\text {sat }}$ [29]. Substituting Eq. (8) into Eq. (7) yields the expression of the universal function of Eq. (6) in the case of

$$
T_{\text {eff }}=\kappa Q_{\text {sat }} .
$$

The gluon saturation is physics of the intermediate energy scale $Q_{\text {sat }}$, while GS observed in the final state is physics of the energy scale $T_{\text {eff }}$ which is much lower than $Q_{\text {sat }}$. Therefore, the parameter $\kappa$ in Eq. (9) [or equivalently Eq. (6)] may have a physical meaning of a linkage between two energy scales of $Q_{\text {sat }}$ and $T_{\text {eff }}$. Before closing Sec. II, let us check $S_{T}$ and $Q_{\text {sat }}$ obtained here. By integrating Eq. (7), we obtain the average multiplicity density [43],

$$
\left\langle\frac{d N_{\mathrm{ch}}}{d y}\right\rangle=\frac{2 \pi S_{T}\left[\kappa Q_{\mathrm{sat}}\right]^{2}}{(2-q)(3-2 q)}=\frac{3.76}{(\mathrm{GeV} / c)^{2}} Q_{\mathrm{sat}}^{2},
$$

which gives $3.68,4.63$, and 5.50 for $W=0.90,2.76$, and $7.00 \mathrm{TeV}$, respectively. These values should be compared with values obtained by experiments [44], i.e., $3.75_{-0.05}^{+0.06}, 4.76_{-0.07}^{+0.08}$, $5.98_{-0.07}^{+0.09}$ for $\sqrt{s}=0.90,2.76$, and $7.00 \mathrm{TeV}$, respectively.

\section{GS FOR SEMI-INCLUSIVE $p_{T}$ DISTRIBUTION}

\section{A. Extraction of saturation momentum scale}

In this section, we will extract the multiplicity dependence of saturation momentum $Q_{\text {sat }}$ from the semi-inclusive spectrum observed. Our central assumption is that the semiinclusive $p_{T}$ distribution scales to the same universal function $\mathcal{F}(\tau)$ as the inclusive one [i.e., Eq. (6) with $q=1.134$, $\kappa=0.1293$, and $\lambda=0.22]$, providing that the appropriate $Q_{\text {sat }}\left(W^{*}\right)$ is used. Since $S_{T}$ in Eq. (3c) now depends on the multiplicity, we require GS for the semi-inclusive spectra as shown by Eq. (5a) with (5b) in Sec. I,

$$
\frac{1}{S_{T}^{*}} \frac{1}{2 \pi p_{T}} \frac{d^{2} n_{\mathrm{ch}}}{d p_{T} d y}=\mathcal{F}(\tau)
$$

and

$$
\tau^{1 /(2+\lambda)}=\frac{p_{T}}{Q_{\mathrm{sat}}\left(W^{*}\right)}
$$

These two parameters, $W^{*}$ and $S_{T}^{*}$, are determined by fitting to the experimental data on the semi-inclusive $p_{T}$ distribution. Note that, in this case, Eq. (4) should be modified as

$$
\frac{d n_{\mathrm{ch}}}{d y} \propto S_{T}^{*} Q_{\mathrm{sat}}^{2}\left(W^{*}\right)
$$




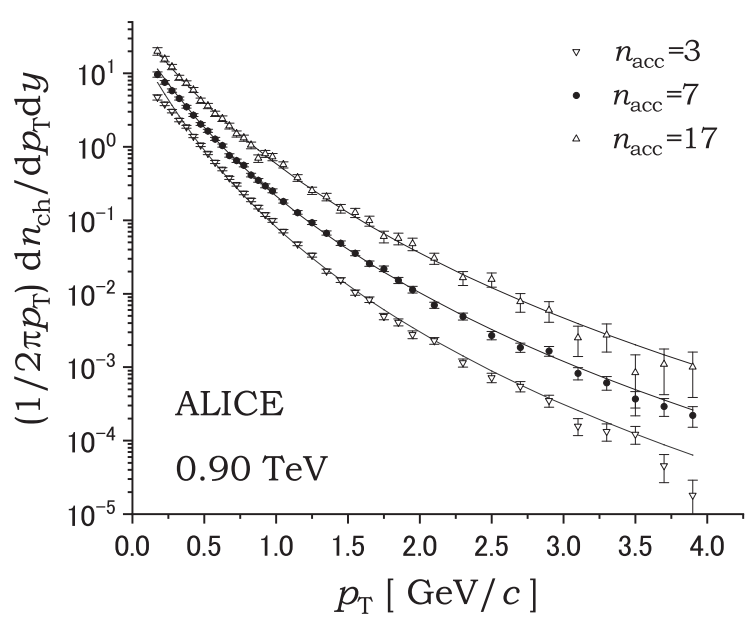

FIG. 3. Fit results of $S_{T}^{*} \mathcal{F}$ [solid curves; see Eqs. (5a) and (5b)] to $p_{T}$ spectra with $n_{\text {acc }}=3,7$, and 17 in $p p$ collisions at energy $0.90 \mathrm{TeV}$ observed by the ALICE Collaboration [45]. The pseudorapidity range is $-0.8 \leqslant \eta \leqslant+0.8$.

Since the universal function in Eq. (5a) is the same as that in Eq. (3c), the proportionality constants in Eqs. (4) and (11) are equal. Therefore, using Eq. (3b), the ratio of $W^{*}$ to $W$ is given by

$$
\begin{aligned}
\frac{W^{*}}{W} & =\left[\frac{S_{T} /\left\langle\frac{d N_{\text {ch }}}{d y}\right\rangle}{S_{T}^{*} / \frac{d n_{\text {ch }}}{d y}}\right]^{\frac{2+\lambda}{2 \lambda}} \\
& = \begin{cases}{\left[0.23\left(\mathrm{fm}^{2}\right) / s_{T}^{*}\right]^{5.05}} & (W=0.90 \mathrm{TeV}) \\
{\left[0.18\left(\mathrm{fm}^{2}\right) / s_{T}^{*}\right]^{5.05}} & (W=2.76 \mathrm{TeV}), \\
{\left[0.16\left(\mathrm{fm}^{2}\right) / s_{T}^{*}\right]^{5.05}} & (W=7.00 \mathrm{TeV})\end{cases}
\end{aligned}
$$

where $s_{T}^{*} \equiv S_{T}^{*} / \frac{d n_{\text {ch }}}{d y}$. As can be seen from Eq. (12), $W^{*}$ and $S_{T}^{*}$ are not independent parameters. Hence, whether $W^{*} / W$ becomes larger or smaller than unity depends on whether a cross-sectional area per gluon $s_{T}^{*}$ in the semi-inclusive distribution is larger or smaller than that in the inclusive distribution. Even if $W^{*}$ has a value greater than $W$, it does not mean an unphysical situation.

In order to determine the multiplicity dependence of $W^{*}$ in Eq. (5b), we fit Eq. (5a) to $p_{T}$ spectra at energy $0.90 \mathrm{TeV}$ for the accepted number of charged particles $n_{\text {acc }}=3,7$, and 17 observed by the ALICE Collaboration [45] and at energies $0.90,2.76$, and $7.00 \mathrm{TeV}$ for the average track multiplicity $n_{\text {tracks }}=40,63,75,98,120$, and 131 observed by the CMS Collaboration [39]. Figures 3 and 4 show the results of fitting with $S_{T}^{*} \mathcal{F}$ to the ALICE and CMS data, respectively. In addition, Table I shows the values of $W^{*}$ (multiplied by $x_{0}$ ) and effective radius $R_{T} \equiv \sqrt{S_{T}^{*} / \pi}$ obtained by the fit. Table I also shows the value of $Q_{\text {sat }}\left(W^{*}\right)$ and the minimum value of $\chi^{2}$ (denoting by $\chi_{\min }^{2}$ ) in each fitting. As shown in Fig. 5, we confirm that the semi-inclusive transverse-momentum spectra depicted in Figs. 3 and 4 scale in terms of the scaling variable $\tau$ of Eq. (5b). Note that the solid curve (the universal function $\mathcal{F}$ ) in Fig. 5 is exactly the same as that obtained in the inclusive distribution in Fig. 1 . We also show $Q_{\text {sat }}$ and $R_{T}$ as functions of $d n_{\mathrm{ch}} / d y$ in Fig. 6. It is found that $Q_{\mathrm{sat}}\left(W^{*}\right)$ and $R_{T}$ are proportional to $\left(d n_{\mathrm{ch}} / d y\right)^{1 / 6}$ and $\left(d n_{\mathrm{ch}} / d y\right)^{1 / 3}$,

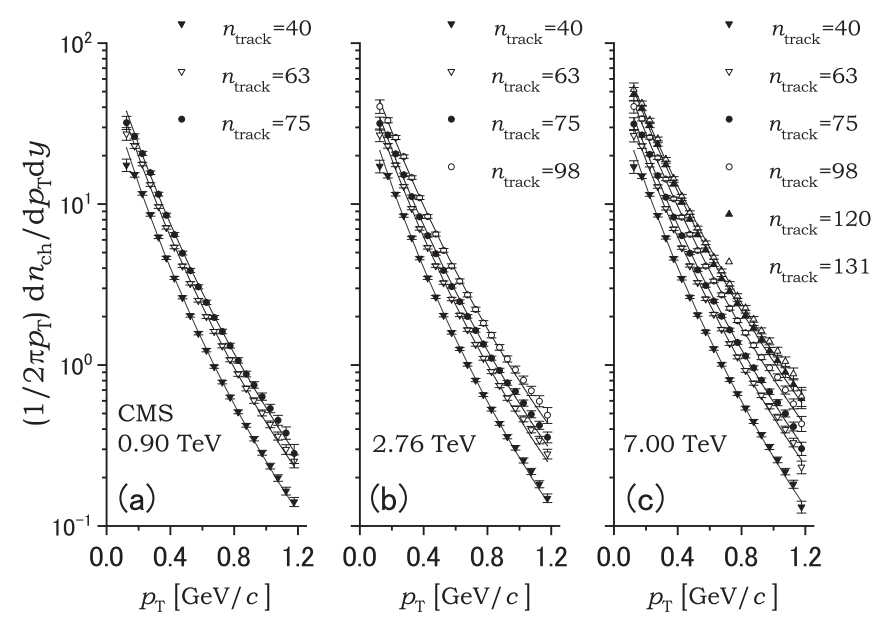

FIG. 4. The same as Fig. 3 but to data on $p_{T}$ spectra for multiplicity selections with $n_{\text {tracks }}=40 \sim 131$ in $p p$ collisions at energies (a) $0.90 \mathrm{TeV}$, (b) $2.76 \mathrm{TeV}$, and (c) $7.00 \mathrm{TeV}$ observed by the CMS Collaboration [39]. The pseudorapidity range is $-2.4 \leqslant \eta \leqslant+2.4$.

respectively. The curves depicted by broken lines in the left panel (for $Q_{\text {sat }}$ ) and the right panel (for $R_{T}$ ) of Fig. 6 are given by

$$
\begin{gathered}
\frac{Q_{\mathrm{sat}}}{(\mathrm{GeV} / c)}= \begin{cases}0.232+0.532\left(\frac{d n_{\mathrm{ch}}}{d y}\right)^{\frac{1}{6}} & (W=0.90 \mathrm{TeV}) \\
0.049+0.669\left(\frac{d n_{\mathrm{ch}}}{d y}\right)^{\frac{1}{6}} & (W=2.76 \mathrm{TeV}), \\
0.031+0.688\left(\frac{d n_{\mathrm{ch}}}{d y}\right)^{\frac{1}{6}} & (W=7.00 \mathrm{TeV})\end{cases} \\
\frac{R_{T}}{(\mathrm{fm})}= \begin{cases}0.039+0.400\left(\frac{d n_{\mathrm{ch}}}{d y}\right)^{\frac{1}{3}} & (W=0.90 \mathrm{TeV}) \\
0.006+0.396\left(\frac{d n_{\mathrm{ch}}}{d y}\right)^{\frac{1}{3}} & (W=2.76 \mathrm{TeV}) . \\
0.006+0.392\left(\frac{d n_{\mathrm{ch}}}{d y}\right)^{\frac{1}{3}} & (W=7.00 \mathrm{TeV})\end{cases}
\end{gathered}
$$

These $d n_{\mathrm{ch}} / d y$ dependencies are consistent with Eq. (11) when $d n_{\mathrm{ch}} / d y$ is sufficiently large and the constant term can be ignored. Here it is interesting to find a particle number density $d n_{\mathrm{ch}} / d y$ to give $W^{*}\left(d n_{\mathrm{ch}} / d y\right)=W$. Using Eqs. (12) and (13b), we can evaluate $s_{T}^{*}$ that satisfies $W^{*} / W=1$. For simplicity, ignoring the constant term of Eq. (13b), we obtain $W^{*}=W$ when $d n_{\mathrm{ch}} / d y=10.4,20.5$, and 27.4 for $0.90,2.76$, and $7.00 \mathrm{TeV}$, respectively. In fact, for CMS event classes with $n_{\text {tracks }}=63,98$, and 131 in $|\Delta \eta|<2.4$ at $W=0.90,2.76$, and 7.00 TeV, respectively, it can be seen from Table I that $W^{*}>W$ is realized.

\section{B. Mean transverse momentum}

Next we turn our attention to the average transverse momentum $\left\langle p_{T}\right\rangle$ obtained from the semi-inclusive distributions. The energy-dependent saturation momentum $Q_{\text {sat }}\left(W^{*}\right)$ should be proportional to $\left\langle p_{T}\right\rangle$ in a GS framework [43]. As seen in the left panel of Fig. 7, $d n_{\mathrm{ch}} / d y$ dependencies of $\left\langle p_{T}\right\rangle$ at 0.90, 2.76, and 7.00 TeV observed by ALICE [46] and CMS [39] do not show scaling behavior in terms of $d n_{\mathrm{ch}} / d y$. However, since GS holds for the semi-inclusive distributions, one 
TABLE I. The values of $x^{0} W^{*}, Q_{\text {sat }}, R_{T}$, and minimum chi-squared $\chi_{\min }^{2}$ obtained from the fitting to the semi-inclusive transversemomentum distribution observed by ALICE [45] with multiplicity class $n_{\text {acc }}=3,7,17$ (accepted number of charged particles per inelastic event in the range $|\eta|<0.8$ ) and by CMS [39] with multiplicity class $n_{\text {tracks }}=40,63,75,98,120$, and 131 (average number of true tracks multiplicity in the range $|\eta|<2.4)$. For assignment from $n_{\text {acc }}$ to $\left\langle n_{\mathrm{ch}}\right\rangle$ in ALICE data, we use results presented in Table 2 of Ref. [45]. The particle densities $d n_{\mathrm{ch}} / d y$ at central rapidity region are estimated by $\left\langle n_{\mathrm{ch}}\right\rangle / \Delta \eta$ for simplicity. $X_{-b}^{+a}$ denotes $X\left(=x_{0} W^{*}, Q_{\mathrm{sat}}, R_{T}\right)$ giving $\chi_{\text {min }}^{2}$ and $a, b$ mean a boundary $X-b \leqq X \leqq X+a$ giving $\chi^{2}=1.5 \chi_{\min }^{2}$.

\begin{tabular}{|c|c|c|c|c|c|c|}
\hline$\sqrt{s}(\mathrm{TeV})$ & $n_{\text {acc }}$ & $\left\langle n_{\mathrm{ch}}\right\rangle / \Delta \eta$ & $x_{0} W^{*}(\mathrm{GeV})$ & $Q_{\text {sat }}(\mathrm{GeV} / c)$ & $R_{T}(\mathrm{fm})$ & $\chi_{\min }^{2} / \mathrm{dof}$ \\
\hline 0.90 & 7 & $10.0 / 1.6$ & $1.39_{-0.43}^{+0.60}$ & $1.03_{-0.04}^{+0.04}$ & $0.69_{-0.04}^{+0.05}$ & $20.5 / 33$ \\
\hline$\sqrt{s}(\mathrm{TeV})$ & $n_{\text {tracks }}$ & $\left\langle n_{\mathrm{ch}}\right\rangle / \Delta \eta$ & $x_{0} W^{*}(\mathrm{GeV})$ & $Q_{\text {sat }}(\mathrm{GeV} / c)$ & $R_{T}(\mathrm{fm})$ & $\chi_{\min }^{2} /$ dof \\
\hline 0.90 & 40 & $40 / 4.8$ & $0.77_{-0.14}^{+0.16}$ & $0.97_{-0.02}^{+0.02}$ & $0.84_{-0.02}^{+0.04}$ & $10.5 / 18$ \\
\hline 0.90 & 75 & $75 / 4.8$ & $1.72_{-0.45}^{+0.22}$ & $1.06_{-0.03}^{+0.04}$ & $1.05_{-0.05}^{+0.05}$ & $17.6 / 18$ \\
\hline 2.76 & 40 & $40 / 4.8$ & $1.05_{-0.24}^{+0.33}$ & $1.00_{-0.03}^{+0.03}$ & $0.81_{-0.03}^{+0.04}$ & $19.8 / 18$ \\
\hline 2.76 & 63 & $63 / 4.8$ & $2.23_{-0.80}^{+0.15}$ & $1.08_{-0.05}^{+0.05}$ & $0.93_{-0.05}^{+0.03}$ & $41.0 / 18$ \\
\hline 2.76 & 75 & $75 / 4.8$ & $\begin{array}{r}2.92_{-1.17}^{+0.70} \\
\end{array}$ & $1.11_{-0.06}^{+0.05}$ & $0.99_{-0.06}^{+0.07}$ & $46.4 / 18$ \\
\hline 2.76 & 98 & $98 / 4.8$ & $3.94_{-1.54}^{+2.24}$ & $1.15_{-0.05}^{+0.05}$ & $1.10_{-0.06}^{+0.07}$ & $32.4 / 18$ \\
\hline 7.00 & 98 & $98 / 4.8$ & $4.95_{-2.00}^{+3.20}$ & $1.17_{-0.06}^{+0.06}$ & $1.07_{-0.07}^{+0.07}$ & $47.1 / 18$ \\
\hline 7.00 & 120 & $120 / 4.8$ & $6.25_{-2.17}^{+3.92}$ & $1.20_{-0.05}^{+0.06}$ & $1.17_{-0.07}^{+0.07}$ & $30.1 / 18$ \\
\hline 7.00 & 131 & $131 / 4.8$ & $8.25_{-3.25}^{+5.67}$ & $1.23_{-0.06}^{+0.07}$ & $1.18_{-0.07}^{+0.07}$ & $35.6 / 18$ \\
\hline
\end{tabular}

expects that $\left\langle p_{T}\right\rangle$ is linearly proportional to $Q_{\text {sat }}\left(W^{*}\right)$ and those data lie on a straight line regardless of the colliding energy $W$. Figure 7 shows results of the conversion of the $d n_{\mathrm{ch}} / d y$ dependence of $\left\langle p_{T}\right\rangle$ on the left panel into the

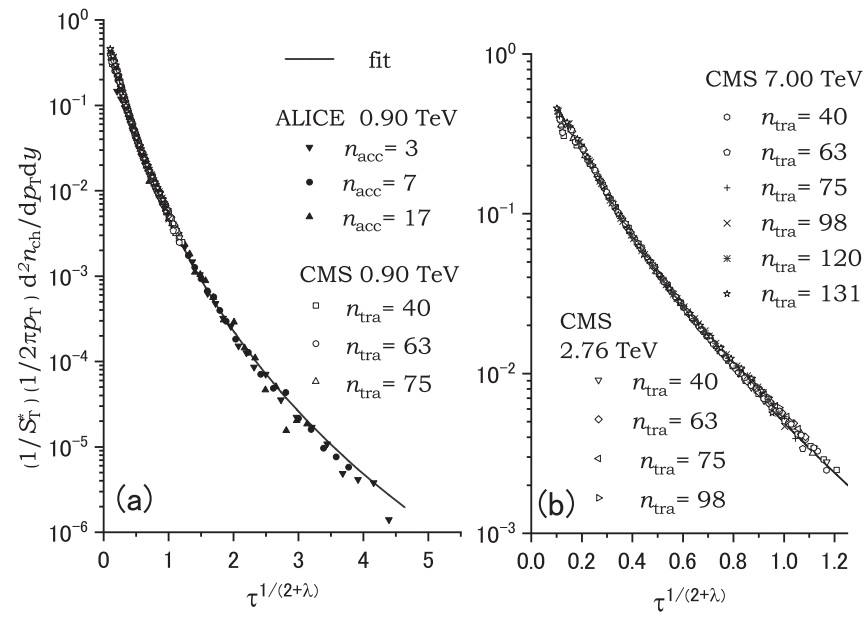

FIG. 5. Geometrical scaling of the semi-inclusive transversemomentum spectra in terms of the scaling variable $\tau$ defined by Eq. (5b) for (a) $0<\tau<5.0$ and (b) the same as (a) but $0<\tau<1.2$. The experimental data are observed by ALICE [45] (open symbols) with multiplicity class $n_{\text {acc }}=3,7,17$ and by CMS [39] (closed symbols) with multiplicity class $n_{\text {tracks }}=40,63,75,98,120$, and 131. dependence of $Q_{\text {sat }}\left(W^{*}\right)$ on the right panel. The difference in scaling curves between ALICE and CMS seems to be due to differences in the acceptance employed. Thus, the behavior of GS is observed not only in the inclusive distributions but also in the semi-inclusive distributions in high-energy $p p$ collisions.
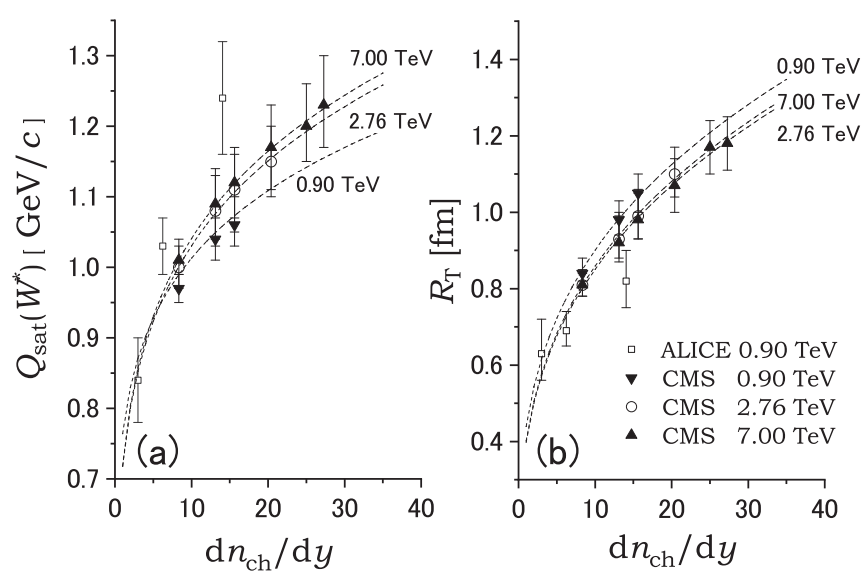

FIG. 6. (a) The energy-dependent saturation momentum $Q_{\text {sat }}\left(W^{*}\right)$ and (b) effective interaction radius $R_{T}$ extracted from the semi-inclusive transverse spectra for $p p$ collisions at energy 0.90 , 2.76 , and 7.00 TeV. The range of $\chi^{2}<1.5 \chi_{\min }^{2}$ is represented as error bars. The dashed curves are fit results (see Eqs. (13a) and (13b)]. 

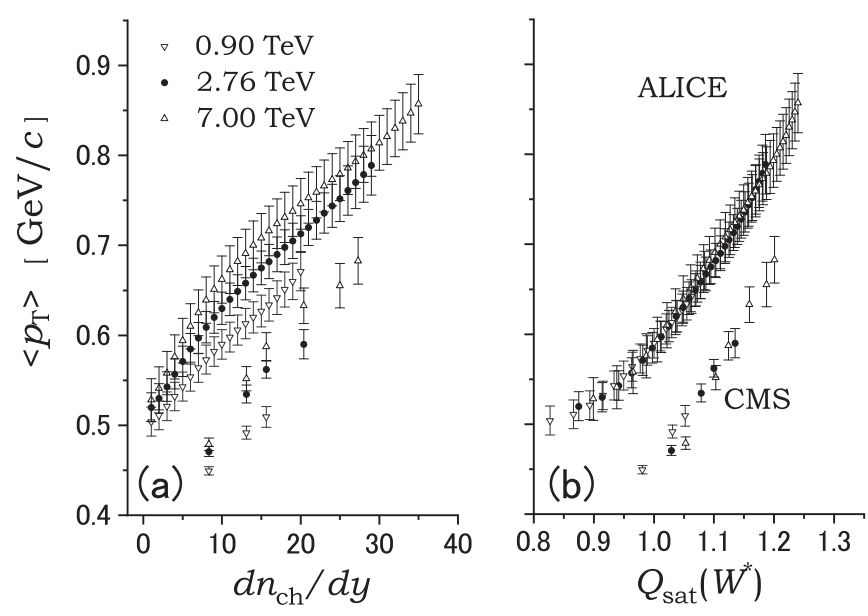

FIG. 7. Average transverse momentum $\left\langle p_{T}\right\rangle$ of $p p$ collisions observed by the ALICE Collaboration [46] and CMS Collaboration [39] as a function of (a) $d n_{\mathrm{ch}} / d y$ and (b) a function of $Q_{\text {sat }}$ (right panel). Since ALICE and CMS select their events with different acceptance, different scaling functions appear.

\section{Normalized fluctuation measure of transverse momentum}

A prominent scaling behavior emerges in event-by-event mean $p_{T}$ fluctuations in $p p$ collisions at LHC energies [47-49]. In our previous work [43], we studied it focusing only on the energy $W=0.90 \mathrm{TeV}$, and we did not discuss the GS behavior by extending the analysis to other energies. In this article, we analyze data on transverse-momentum fluctuations observed at $\sqrt{s}=0.90,2.76,7.00 \mathrm{TeV}$ using $Q_{\text {sat }}\left(W^{*}\right)$ and $S_{T}^{*}$ without changing the basic idea of the model proposed in Ref. [43]. The fluctuation measure is essentially a two-particle distribution as defined below,

$C_{m}=\frac{\int d^{2} \mathbf{p}_{\mathrm{T}_{1}} \int d^{2} \mathbf{p}_{\mathrm{T}_{2}}}{m(m-1)} \frac{d^{4} n_{\mathrm{ch}}}{d \mathbf{p}_{\mathrm{T}_{1}}^{2} d \mathbf{p}_{\mathrm{T}_{2}}^{2}}\left(p_{\mathrm{T}_{1}}-\left\langle p_{T}\right\rangle\right)\left(p_{\mathrm{T}_{2}}-\left\langle p_{T}\right\rangle\right)$,

where $m=\frac{d n_{\mathrm{ch}}}{d \eta} \times|\Delta \eta|$ is the multiplicity in the pseudorapidity window $|\Delta \eta|$. Since the universal function of GS is essentially one-particle distribution, the two-particle correlation function [50] as shown below is required to obtain the two-particle distribution in Eq. (14),

$$
C\left(\mathbf{p}_{\mathrm{T}_{1}}, \mathbf{p}_{\mathrm{T}_{2}}\right) \equiv \frac{d^{4} n_{\mathrm{ch}}}{d \mathbf{p}_{\mathrm{T}_{1}}^{2} d \mathbf{p}_{\mathrm{T}_{2}}^{2}} / \frac{d^{2} n_{\mathrm{ch}}}{d \mathbf{p}_{\mathrm{T}_{1}}^{2}} \frac{d^{2} n_{\mathrm{ch}}}{d \mathbf{p}_{\mathrm{T}_{2}}^{2}} .
$$

It is known that a gluon two-particle correlation function takes the following simple geometrical form in the CGC/Glasma framework [51-53]:

$$
C_{\mathrm{GFT}}\left(\mathbf{p}_{\mathrm{T}_{1}}, \mathbf{p}_{\mathrm{T}_{2}}\right)=1+\frac{\kappa_{2}}{S_{T} Q_{\mathrm{sat}}^{2}},
$$

where $\kappa_{2}$ is a nonperturbative constant, and the evaluation of this constant is a challenging problem in theoretical physics. On the other hand, we consider an extreme model in which the correlation in momentum space between gluons is inherited to that between hadrons in the final state. Since the transverse size of color flux tubes stretching between the

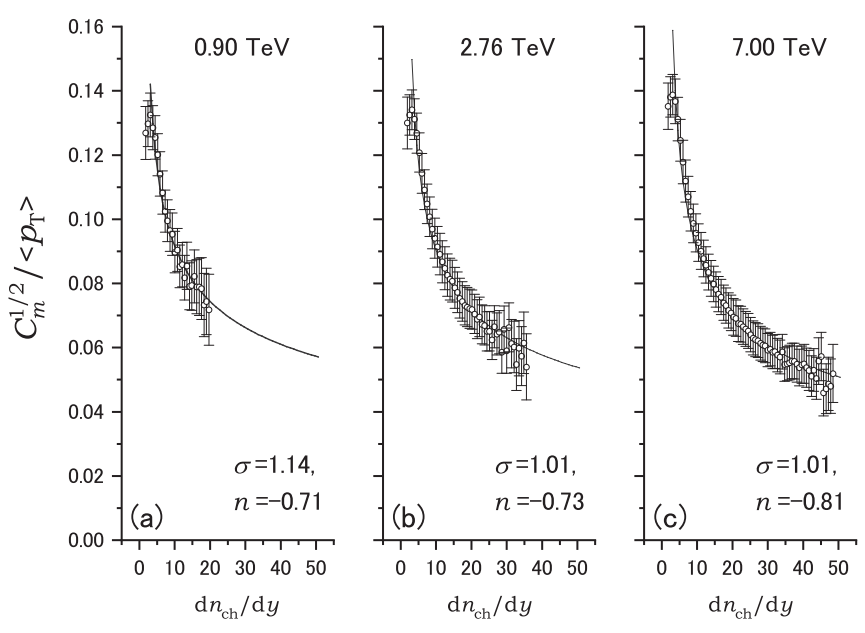

FIG. 8. Experimental data on event-by-event mean transversemomentum fluctuation [47] and fit results of our model [Eq. (17)] to the data observed at (a) $\sqrt{s}=0.90 \mathrm{TeV}$, (b) $2.76 \mathrm{TeV}$, and (c) $7.00 \mathrm{TeV}$. The pseudorapidity window is $|\Delta \eta|=0.8$. Table II shows the values of the parameters that give the least chi-square for the fitting of $\sqrt{C_{m}} /\left\langle p_{T}\right\rangle$. We have excluded two small $d N_{\mathrm{ch}} / d y(=1.8$ and 2.4) data points from the fits.

receding protons is expected to be of order in $1 / Q_{\text {sat }}$, one may write the following correlation function commonly found in Bose-Einstein correlation (BEC) analysis:

$$
C\left(\mathbf{p}_{\mathrm{T}_{1}}, \mathbf{p}_{\mathrm{T}_{2}}\right)=1+\left(S_{T}^{*}\left[\kappa Q_{\mathrm{sat}}\right]^{2}\right)^{n} \exp \left[-\frac{\left(\mathbf{p}_{\mathrm{T}_{1}}-\mathbf{p}_{\mathrm{T}_{2}}\right)^{2}}{\sigma\left[\kappa Q_{\mathrm{sat}}\right]^{2}}\right],
$$

where $n$ and $\sigma$ are model parameters. Here $\kappa$ is the parameter that appears in the universal function Eq. (6) which connects intermediate energy scale $Q_{\text {sat }}$ and hadronization energy scale $T_{\text {eff }}$. Since $\kappa$ and $Q_{\text {sat }}$ always appear together in the inclusive distribution, there must also be such a property in the twoparticle distribution in Eq. (17). Note also that the term $S_{T}^{*} Q_{\text {sat }}^{2}$ in Eq. (17) is proportional to the number of flux tubes [23], especially when $n=-1$; it can be interpreted as chaoticity of the BEC effect [43]. Another parameter $\sigma$ is for adjusting the size of the flux tube. When $\sigma \approx 1$, it means that the size of the color flux tube is expanded by about $1 / \kappa \approx 7.7$ times in the transverse direction and the source size scale is the inverse of the temperature of the system $\sim 1 / T_{\text {eff }}$. As seen in Fig. 8 , ALICE observed a normalized fluctuation measure $\sqrt{C_{m}} /\left\langle p_{T}\right\rangle$ at $W=0.90,2.76$, and $7.00 \mathrm{TeV}$, and they found almost no energy dependence in them. Our model, based on GS, easily explains the reason the measure $\sqrt{C_{m}} /\left\langle p_{T}\right\rangle$ hardly depends on the collision energy: i.e., by noting that $p_{T}=Q_{\text {sat }} \tau^{1 /(2+\lambda)}$, $\left\langle p_{T}\right\rangle \propto Q_{\text {sat }}$, and $m \propto S_{T}^{*} Q_{\text {sat }}^{2}$, one can represent the measure as a function of the scaling variable $\tau$ except for the term $S_{T}^{*} Q_{\text {sat }}^{2}$ in the correlation function Eq. (17). However, as shown by Eqs. (13a) and (13b), the energy dependence of both $Q_{\text {sat }}$ and $S_{T}^{*}$ are considerably small. Moreover, recall that $S_{T}^{*} Q_{\text {sat }}^{2}$ is the number of color flux tubes. Since the gluon in the incident proton is saturated regardless of the energy, it is natural that the energy dependence of this factor is small. Therefore, 
TABLE II. The best fit values of the parameters $\sigma$ and $n$ to the experimental data on the event-by-event fluctuation of mean $p_{T}$ observed by the ALICE Collaboration [47] and results of evaluation of $\kappa_{2}$ by Eq. (18).

\begin{tabular}{lcccc}
\hline \hline$\sqrt{s}(\mathrm{TeV})$ & $\sigma$ & $n$ & $\chi_{\min }^{2} / \mathrm{dof}$ & $\kappa_{2}$ \\
\hline 0.90 & 1.14 & -0.71 & $4.93 / 23$ & 1.79 \\
2.76 & 1.01 & -0.73 & $14.0 / 45$ & 1.14 \\
7.00 & 1.01 & -0.81 & $20.4 / 64$ & 1.14 \\
\hline \hline
\end{tabular}

it is explained that $\sqrt{C_{m}} /\left\langle p_{T}\right\rangle$ is almost independently of the colliding energy $W$ in our model. The fit results to the experimental data of Eq. (17) are shown by solid lines in Fig. 8. We also show values of the parameter both $\sigma$ and $n$ giving $\chi_{\min }^{2}$ in Table II. The values of $n$ obtained by the fits are from -0.71 to -0.81 , which are larger than -1 , but Eq. (17) can be compared with the Eq. (16) in the Glasma framework. Evaluating the typical momentum scale of BEC as $\left|\mathbf{p}_{\mathrm{T}_{1}}-\mathbf{p}_{\mathrm{T}_{2}}\right|^{2} \sim\left[2 \kappa Q_{\text {sat }}\right]^{2} \approx[200 \mathrm{MeV}]^{2}$, the comparison leads us to a rough estimation of $\kappa_{2}$ as follows:

$$
\kappa_{2} \sim \frac{1}{\kappa^{2}} \exp \left(-\frac{4}{\sigma}\right) \text {. }
$$

Table II also shows the values of $\kappa_{2}$ evaluated by Eq. (18). Since there are considerable variations in the extracted values of $\kappa_{2}$ from experimental data based on the Glasma framework, its value is not known to be as accurate as an order of 1 [52]. It is interesting to note that the values of $\kappa_{2}$ extracted from our model are comparable to the estimation by the Glasma framework, although the picture for particle correlation of each other is different.

\section{SUMMARY AND CONCLUDING REMARKS}

In this article, we have phenomenologically investigated multiplicity dependence on the gluon saturation momentum in high-energy $p p$ collisions. This result makes it possible to classify events by energy-dependent saturation momentum $Q_{\text {sat }}\left(W^{*}\right)$, which in turn can provide a new research approach to high-energy multiparticle production.

If the local parton-hadron duality hypothesis is correct, then $Q_{\text {sat }}\left(W^{*}\right)$ must link to observables in the final state of the charged hadrons. In order to extract $Q_{\text {sat }}\left(W^{*}\right)$ that governs the multiplicity of the final states, we assumed the semiinclusive transverse-momentum spectra exhibit geometrical scaling behavior independently of its fixed multiplicity and its colliding energy. Furthermore, the universal function is assumed to be the same as that of the inclusive distribution. Through the effective energy $W^{*}$ defined by Eq. (5b), we determined $Q_{\text {sat }}\left(W^{*}\right)$ for the semi-inclusive distributions. We have shown that the transverse-momentum distribution of various multiplicity class at $\sqrt{s}=0.90,2.76$, and $7.00 \mathrm{TeV}$ do scale in terms of the scaling variable $\tau^{1 /(2+\lambda)}=p_{T} / Q_{\text {sat }}\left(W^{*}\right)$. We have also confirmed that $Q_{\text {sat }}\left(W^{*}\right)$ dependence on the average transverse momentum also scales to a linear function of $Q_{\text {sat }}\left(W^{*}\right)$, which is consistent with the behavior expected from GS.

It is meaningful to note works by Korus and Mrówczyński $[54,55]$ and to compare with the model we have proposed. Korus and Mrówczyński have introduced a multiplicitydependent temperature and related the nontrivial behavior of fluctuations in the transverse momentum to that in the multiplicity distribution. In our model, on the other hand, the energy-dependent saturation momentum $Q_{\text {sat }}\left(W^{*}\right)$ is related to the multiplicity of the final state via the effective energy $W^{*}$ and is also related to the temperature evaluated from the semi-inclusive spectra by Eq. (9). As for fluctuation of transverse momentum, Korus and Mrówczyński argue that the reason for this is that the fluctuation in the multiplicity distribution is almost independent of energy. In fact, the normalized $q$-moment values of $C_{2}, C_{3}, C_{4}$ for the multiplicity distribution in the central rapidity region $|\eta|<0.5$ are almost independent of the collision energy $[44,56]$. On the other hand, in our model, the reason there is almost no dependence on collision energy in the fluctuation measure $\sqrt{C_{m}} /\left\langle p_{T}\right\rangle$ is that the energy dependencies on $Q_{\text {sat }}\left(W^{*}\right)$ and $S_{T}$ are considerably small (see Fig. 6) in addition to the fact that the semi-inclusive transverse-momentum spectrum shows the behavior of geometrical scaling.

In this article, we thought that the two-particle BoseEinstein correlation between identical gluons produced from color flux tubes could explain the experimental results of the fluctuation measure. The measure $\sqrt{C_{m}} /\left\langle p_{T}\right\rangle$ can be fitted by Eq. (17) nicely, in which the correlation between gluons is considered to remain between charged particles after hadronization. Comparing Eq. (16) with our model Eq. (17) we can estimate the value of the nonperturbative constant of the gluon correlation function $\kappa_{2}$. If a typical value for $\left|\mathbf{p}_{\mathrm{T}_{1}}-\mathbf{p}_{\mathrm{T}_{2}}\right|$ in Eq. (20) as $200 \mathrm{MeV} / c$ is adopted, one obtain $\kappa_{2}=1.4-1.8$. It is interesting to extract $Q_{\text {sat }}$ from other reaction such as $p A$ [57] and $A A$ [58] collisions and to discuss the relationship between the fluctuation of multiplicity and that of the saturation momentum. However, we plan to investigate those issues at some other opportunity.

\section{ACKNOWLEDGMENTS}

We acknowledge stimulating discussions with Grzegorz Wilk concerning $q$ scaling in high-energy production processes and the effective energy. We also thank the anonymous referee for thorough comments which have greatly improved the manuscript.
[1] R. Preghenella, Small systems at the LHC, EPJ Web Conf. 171, 11003 (2018).

[2] V. Khachatryan et al. (CMS Collaboration), J. High Energy Phys. 09 (2010) 091.
[3] V. Khachatryan et al. (CMS Collaboration), Phys. Lett. B 765, 193 (2017).

[4] K. Dusling, W. Li, and B. Schenke, Int. J. Mod. Phys. E 25, 1630002 (2016). 
[5] J. L. Nagle and W. A. Zajc, Annu. Rev. Nucl. Part. Sci. 68, 211 (2018).

[6] J. Adam et al. (ALICE Collaboration), Nat. Phys. 13, 535 (2017).

[7] D. Kharzeev and M. Nardi, Phys. Lett. B 507, 121 (2001).

[8] L. McLerran and M. Praszalowicz, Acta Phys. Pol. B 41, 1917 (2010).

[9] J. P. Blaizot and A. H. Mueller, Nucl. Phys. B 289, 847 (1987).

[10] L. V. Gribov, E. M. Levin, and M. G. Ryskin, Phys. Rep. 100, 1 (1983).

[11] A. H. Mueller and J.-w. Qiu, Nucl. Phys. B 268, 427 (1986).

[12] D. Kharzeev and E. Levin, Phys. Lett. B 523, 79 (2001).

[13] Y. V. Kovchegov and E. Levin, Quantum Chromodynamics at High Energy, Vol. 33 (Cambridge University Press, Cambridge, 2012).

[14] E. Iancu, A. Leonidov, and L. McLerran, The color glass condensate: An introduction, in QCD perspectives on hot and dense matter, in Proceedings of NATO Advanced Study Institute, Summer School, Cargese, France, August 6-18, 2001 (Kluwer Academic, Dordrecht, Netherlands, 2002), pp. 73-145.

[15] J.-P. Blaizot and F. Gelis, Nucl. Phys. A 750, 148 (2005).

[16] F. Gelis, E. Iancu, J. Jalilian-Marian, and R. Venugopalan, Annu. Rev. Nucl. Part. Sci. 60, 463 (2010).

[17] A. Stasto, Nucl. Phys. A 854, 64 (2011).

[18] J. Jalilian-Marian, Phys. Rev. C 70, 027902 (2004).

[19] L. McLerran, Structure and dynamics of elementary matter, in Proceedings of the NATO Advanced Study Institute (Kluwer Academic, Dordrecht, Netherlands, 2004), pp. 183-205.

[20] E. Iancu, K. Itakura, and L. McLerran, Nucl. Phys. A 721, 293 (2003).

[21] E. Iancu, K. Itakura, and L. McLerran, Nucl. Phys. A 708, 327 (2002).

[22] A. M. Stasto, K. J. Golec-Biernat, and J. Kwiecinski, Phys. Rev. Lett. 86, 596 (2001).

[23] P. Tribedy and R. Venugopalan, Nucl. Phys. A 850, 136 (2011); 859, 185 (2011).

[24] E. Iancu, K. Itakura, and S. Munier, Phys. Lett. B 590, 199 (2004).

[25] E. Gotsman and E. Levin, Eur. Phys. J. C 79, 415 (2019).

[26] S. Munier and R. B. Peschanski, Phys. Rev. Lett. 91, 232001 (2003).

[27] K. J. Golec-Biernat and M. Wusthoff, Phys. Rev. D 59, 014017 (1998).

[28] M. Praszałowicz and A. Francuz, Phys. Rev. D 92, 074036 (2015).

[29] M. Praszalowicz, Phys. Lett. B 727, 461 (2013).

[30] M. Praszalowicz, Phys. Rev. Lett. 106, 142002 (2011).

[31] M. Praszalowicz, Acta Phys. Pol. B 42, 1557 (2011).

[32] V. Khachatryan and M. Praszalowicz, arXiv:1907.03815 [nucl-th].
[33] D. Kharzeev, E. Levin, and M. Nardi, Nucl. Phys. A 747, 609 (2005).

[34] L. McLerran and M. Praszalowicz, Phys. Lett. B 741, 246 (2015).

[35] Y. I. Azimov, Y. L. Dokshitzer, V. A. Khoze, and S. I. Troyan, Z. Phys. C 27, 65 (1985).

[36] T. Kanki, K. Kinoshita, H. Sumiyoshi, and F. Takagi, Prog. Theor. Phys. Suppl. 97A, 1 (1988).

[37] In this paper, the inclusive spectra is denoted by $d^{2} N_{\mathrm{ch}} / d p_{\mathrm{T}} d y$, and the semi-inclusive spectra is denoted by $d^{2} n_{\mathrm{ch}} / d p_{\mathrm{T}} d y$ to distinguish it from the inclusive one.

[38] B. B. Abelev et al. (ALICE Collaboration), Eur. Phys. J. C 73, 2662 (2013).

[39] S. Chatrchyan et al. (CMS Collaboration), Eur. Phys. J. C 72, 2164 (2012).

[40] M. Rybczynski, Z. Wlodarczyk, and G. Wilk, Acta Phys. Polon. Supp. 6, 507 (2013).

[41] M. Rybczynski, Z. Wlodarczyk, and G. Wilk, J. Phys. G 39, 095004 (2012).

[42] L. McLerran, M. Praszalowicz, and B. Schenke, Nucl. Phys. A 916, 210 (2013).

[43] T. Osada and M. Ishihara, J. Phys. G 45, 015104 (2018).

[44] J. Adam et al. (ALICE Collaboration), Eur. Phys. J. C 77, 33 (2017).

[45] K. Aamodt et al. (ALICE Collaboration), Phys. Lett. B 693, 53 (2010).

[46] B. B. Abelev et al. (ALICE Collaboration), Phys. Lett. B 727, 371 (2013).

[47] B. B. Abelev et al. (ALICE Collaboration), Eur. Phys. J. C 74, 3077 (2014).

[48] S. T. Heckel (ALICE Collaboration), Event-by-event mean pT fluctuations in $\mathrm{pp}$ and $\mathrm{Pb}-\mathrm{Pb}$ collisions at the LHC, EPJ Web Conf. 90, 08006 (2015).

[49] S. Heckel (ALICE Collaboration), Quark matter, J. Phys. G 38, 124095 (2011).

[50] S. Gavin, L. McLerran, and G. Moschelli, Phys. Rev. C 79, 051902(R) (2009).

[51] A. Dumitru, F. Gelis, L. McLerran, and R. Venugopalan, Nucl. Phys. A 810, 91 (2008).

[52] T. Lappi, S. Srednyak, and R. Venugopalan, J. High Energy Phys. 01 (2010) 066.

[53] T. Lappi, Prog. Theor. Phys. Suppl. 187, 134 (2011).

[54] R. Korus and S. Mrowczynski, Phys. Rev. C 64, 054906 (2001).

[55] S. Mrowczynski, M. Rybczynski, and Z. Wlodarczyk, Phys. Rev. C 70, 054906 (2004).

[56] V. Khachatryan et al. (CMS Collaboration), J. High Energy Phys. 01 (2011) 079.

[57] L. McLerran and M. Praszalowicz, Ann. Phys. 372, 215 (2016).

[58] C. Andres, A. Moscoso, and C. Pajares, Nucl. Phys. A 901, 14 (2013). 